\title{
Breaking a Masked AES Implementation Using a Deep Learning-based Attack
}

\author{
Daehyeon Bae \\ Division of Computer and Information \\ Engineering \\ Hoseo University \\ Asan, South Korea \\ noeyheadb@gmail.com
}

\author{
Jongbae Hwang \\ Division of Computer and Information \\ Engineering \\ Hoseo University \\ Asan, South Korea \\ qlghd98@gmail.com
}

\author{
Jaecheol $\mathrm{Ha}^{\dagger}$ \\ Division of Computer and Information \\ Engineering \\ Hoseo University \\ Asan, South Korea \\ jcha@hoseo.edu
}

\begin{abstract}
The block cipher AES (Advanced Encryption Standard) is a cryptographic algorithm used to guarantee the confidentiality of a message. A masked implementation of AES is often used to increase resistance against SCA (Side Channel Attacks). This paper presents some deep learning-based attacks for extracting AES secret keys embedded in cryptographic devices. The proposed attack methods represent new approaches to computing the secret key by applying the mask profiling techniques. The MLP (MultiLayer Perceptron) and CNN (Convolutional Neural Network) deep learning models are developed to break the masked AES implementation. Our experimental results show the overwhelming advantages of the novel attack methods when targeting both unmasked and masked implementation of AES.
\end{abstract}

\section{CCS CONCEPTS}

- Security and Privacy $\rightarrow$ Side-channel analysis and countermeasure; •Computing methodologies $\rightarrow$ Neural networks; - Computer systems organization $\rightarrow$ Embedded systems

\section{KEYWORDS}

Side Channel Attack, Power Analysis, AES, Masked Implementation, MLP, CNN

\section{ACM Reference format:}

Daehyeon Bae, Jongbae Hwang and Jaecheol Ha. 2020. Breaking a Masked AES Implementation Using a Deep Learning-based Attack. In Proceedings of International Conference on Intelligent Computing and its Emerging Applications (ICEA'20), December 12-15, 2020, GangWon, Republic of Korea. ACM, New York, NY, USA, 5 pages. https://doi.org/10.1145/ 3440943.3444724

\footnotetext{
${ }^{\dagger}$ Corresponding Author

Permission to make digital or hard copies of part or all of this work for personal or classroom use is granted without fee provided that copies are not made or distributed for profit or commercial advantage and that copies bear this notice and the full citation on the first page. Copyrights for third-party components of this work must be honored. For all other uses, contact the owner/author(s).
}

\section{INTRODUCTION}

To ensure the confidentiality of messages between two communication parties, a block cipher algorithm such as AES (Advanced Encryption Standard)[1] can be used for data encryption. However, the power analysis attack, one of the many SCAs (Side Channel Attacks)[2, 3], has been proposed to allow an adversary to extract secret information from embedded crypto devices. In particular, a SCA $[4,5]$ on a block cipher is well known as a serious threat in which the secret key can be leaked from the physical implementation of encryption algorithms.

To deal with SCAs, several countermeasures have been published. Among them, so-called masking-based countermeasures are often used to provide randomness in the intermediate values. That is, the AES implementation can also be protected through the message masking countermeasure. The Boolean masking method (e.g., the XOR operation) which randomizes the S-Box output is one of the popular countermeasures[6].

The masking can be characterized by the number of random masks per sensitive variables. Therefore, it is possible to provide a $d$-th order countermeasure definition when d masks are applied to a variable. If a random mask is applied to AES implementation, it can provide resistance to first order DPA (Differential Power Analysis) attacks.

In this paper, we propose some deep learning attacks that can recover the secret key using a power analysis attack based on a profiling data set. Since the measured power signal is time-series data with fixed length, the MLP (Multi-Layer Perceptron)[7] and CNN (Convolutional Neural Network)[8] deep learning models are more effective than RNN (Recurrent Neural Network) to break the first order masked AES implementation.

We separate the Deep Learning (DL)-based attack into two steps: the mask recovery and the masked Sbox output recovery step. To

ACM ICEA '20, December 12-15, 2020, GangWon, Republic of Korea (C) 2020 Association for Computing Machinery. ACM ISBN 978-1-4503-8304-2/20/12 _.\$15.00 https://doi.org/10.1145/3440943.3444724 
perform the proposed MLP and CNN attacks, we implement the AES-128 encryption algorithm on an XMEGA128 experimental board[9]. As a result, we can recover the masked AES secret key with $81.0 \%$ accuracy when using the MLP attack with power consumption traces and with $75.4 \%$ accuracy when using the $\mathrm{CNN}$ attack.

\section{OVERVIEW OF SIDE CHANNEL ATTACK}

\subsection{Power Analysis Attack}

Side channel attacks exploit side channel leakages, such as power consumption and electromagnetic emission, from a cryptographic device to extract secret information. These attacks can be classified into two classes; one of these consists of so-called profiled attacks such as the Template Attack (TA)[10], the Stochastic Attack (SA)[11], and ML-based attacks[12-16]. For a profiled attack, the attacker needs to have access to two identical devices: a profiling device and a target device.

The other attack type consists of non-profiled attacks such as Differential Power Analysis (DPA)[2], Correlation Power Analysis (CPA)[3], and Mutual Information Analysis[17]. In a first order attack on a naïve AES implementation having fixed secret key, the adversary collects several side channel traces of Sbox output $\mathrm{S}=\mathrm{Sbox}(\mathrm{d} \bigoplus \mathrm{k})$, and combines key hypotheses with the use of a statistical distinguisher such as Pearson correlation to extract secret information from the side channel traces. Here, $d$ is an input message and $\mathrm{k}$ is a secret key.

Since our focus in this paper is a DL-based side channel attack, which is a kind of ML (Machine Learning)-based method, we need two experimental devices. One is a profiling device to collect training traces and the other is a target device in which the real secret key is embedded.

\subsection{Countermeasure}

A countermeasure to protect cryptographic implementation is to remove the dependency between leakage traces and the sensitive intermediate value using random masks. Boolean masking is commonly used to protect block cipher algorithms such as AES. In Boolean masking, a sensitive intermediate value, for example the Sbox output of AES, is XORed with a mask as follows:

$$
\mathrm{S}=\operatorname{MSbox}\left(\mathrm{d} \oplus \mathrm{m}_{\mathrm{i}} \oplus \mathrm{k}\right)=\operatorname{Sbox}(\mathrm{d} \oplus \mathrm{k}) \oplus \mathrm{m}_{\mathrm{o}} \text {. }
$$

We focus on the above first order masking, where an Sbox operation is protected by a random input mask $\mathrm{S}=\operatorname{MSbox}\left(\mathrm{d} \oplus \mathrm{m}_{\mathrm{i}}\right.$ $\bigoplus \mathrm{k})$. A high order attack targeting an AES implementation with one mask is called a second order attack. Here, the value of $(\mathrm{d} \oplus$ $\mathrm{k})$ is denoted as $\mathrm{x}$. That is, $\mathrm{S}=\operatorname{MSbox}\left(\mathrm{x} \oplus \mathrm{m}_{\mathrm{i}}\right)=\operatorname{Sbox}(\mathrm{x}) \oplus \mathrm{m}_{\mathrm{o}}$. We make MSbox in advance using the relationship between Sbox and MSbox as follows:

$\operatorname{MSbox}\left[\right.$ index $\left.\oplus \mathrm{m}_{\mathrm{i}}\right]=$ Sbox $[$ index $] \oplus \mathrm{m}_{\mathrm{o}}$.

In a second order attack, an adversary needs to combine the trace leakages related to two masks using a combination function such as the absolute difference. Then, a statistical distinguisher is used to extract the secret key from the combined leakage traces.

\section{DEEP LEARING TECHNIQUES}

Deep learning is a branch of Machine Learning that uses the neural network. A neural network is composed of several perceptron units. The DL-based side channel attack has been applied to many cryptographic algorithms such as AES, RSA (Rivest, Shamir, and Adelman) and ECC (Elliptic Curve Cryptography). Data classification using deep learning consists of the following two steps:

- A training/learning phase: The DL method processes the training data set and automatically tunes the network weights to minimize output loss.

- A classification phase: Data whose corresponding label is unknown is classified.

\subsection{MLP}

A MLP is a combination of many perceptron units that are organized in layers as shown in Figure 1. Each perceptron output is connected to each input of the next layer perceptron. A MLP model is composed of an input layer, an output layer, and hidden layers. In the training phase, the weights of the neural network are optimized to minimize the final output loss.

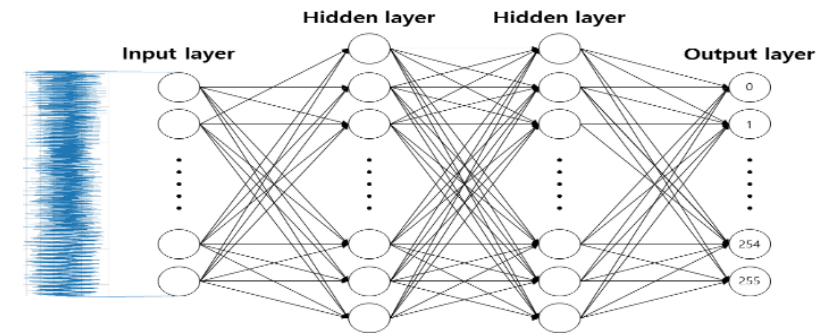

Figure 1: Multi-Layer Perceptron model.

\subsection{CNN}

A CNN is a kind of deep neural network which has two types of layers for feature extraction, as shown in Figure 2: a convolutional layer and a pooling layer. This model has shown particularly excellent results in image recognition tasks. In the convolutional layer, the convolution operations are applied to the input by sliding a set of filters. During the training phase of a CNN, the weights of the filters are tuned in order to minimize the output loss. The pooling layers are used to save the computational demands by reducing the spatial size of the data.

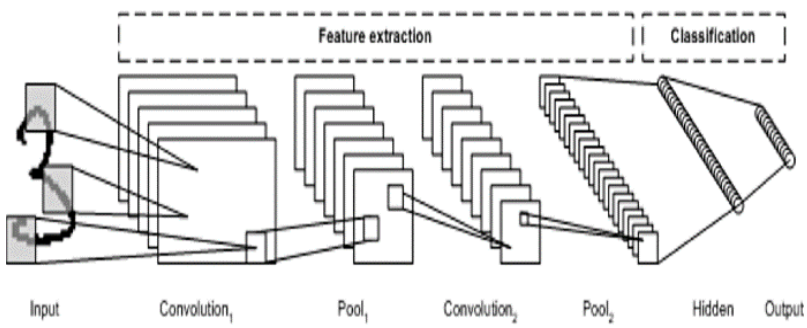

Figure 2: Convolutional Neural Network model. 
Breaking a Masked AES Implementation Using ...

\section{DEEP LEARING-BASED SIDE CHANNEL ATTACK}

In the following section, we compare the efficiencies of our proposed DL-based attacks with those of previous attacks.

\subsection{Experimental Setup}

Since we are dealing with profiled side channel attacks, we assume that an adversary has full control of a training device and the ability to measure the power consumption traces during the profiling phase. The adversary then tries to recover the AES secret key by collecting new power traces from a target device.

For these experiments, we implement an AES-128 encryption algorithm on a XMEGA128 board with a 7.37MHz clock rate. Then, we obtain the dataset of power traces using Chipwhisperer-Lite from NewAE Technology. Here, the dataset consists of $75 \%$ training traces and $25 \%$ validation traces.

\subsection{Attack on Naïve AES Implementation}

The AES-128 algorithm with the 128-bit key size is a block cipher as shown in Figure 3. First, the plaintext composed of 16 bytes is XORed with the initial round key using an AddRoundKey function. The state after initial AddRoundKey, which is a $4 \times 4$ matrix of bytes, is followed by a round transformation composed of the SubBytes, ShiftRows, MixColumns, and AddRoundKey functions. The final round skips the MixColumns operation.

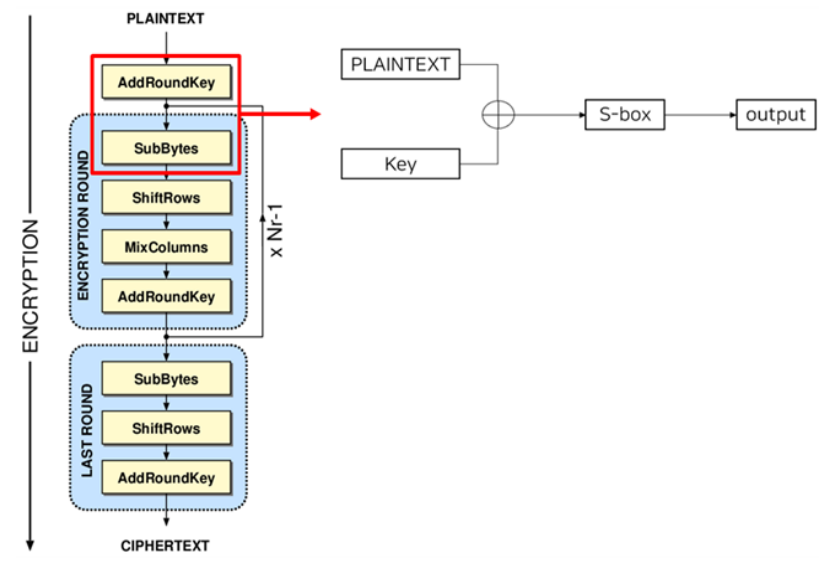

Figure 3: AES encryption algorithm.

During the encryption processing, we assume that the adversary can collect power consumption traces. Figure 4 shows a power trace measured during the round operations with particular magnification of the initial AddRoundKey and SubBytes of the first round. We can see the 16 small power signals at each function operation.

The training data with respect to the Sbox output were profiled. The features of the learning data are the sample values of the power signal, and the label is the output value of Sbox. Therefore, the input layer has the same number of neurons as the number of samples of the measured power trace.
ICEA 2020, December 12-15, 2020, Gangwon, Republic of Korea

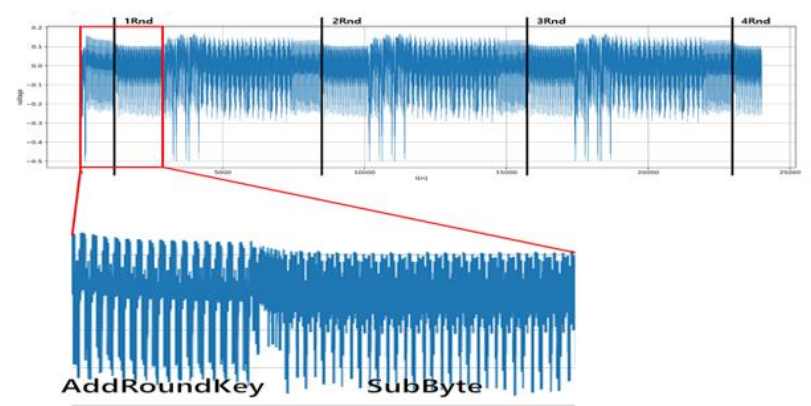

Figure 4: Power consumption trace during AES encryption.

The output layer classifies the input vector to Sbox output and contains 256 neurons for all combinations of output values ranging from 0 to 255 . The byte value of the secret key used in the initial AddRoundKey operation can be recovered (d $\bigoplus$ Inverse Sbox(x)) as shown in Figure 5.

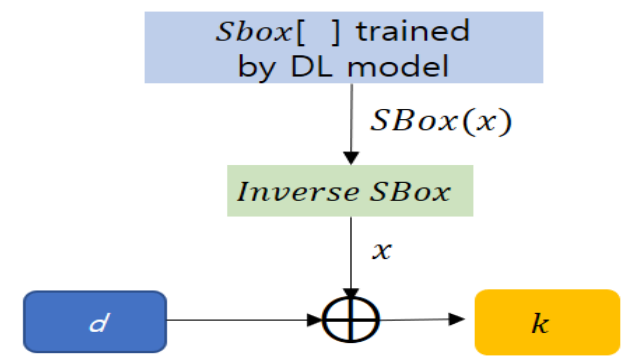

Figure 5: First order attack using Sbox output training.

In the MLP model, we adopt two hidden layers that each have 500 nodes. We collect all 10,000 traces and separate them into 7,500 traces for a training data set and 2,500 traces for a validation data set. The number of input nodes is the same as that of the sample point of a trace. In this experiment, only 100 sample points per trace of Sbox output are used as inputs. Since we need to classify the output of SubBytes, the output has 256 nodes. The cross-entropy is used as a loss function and the Adam function is used as an optimizer.

In the $\mathrm{CNN}$ model, the numbers of input and output nodes are the same as those in the MLP model. Although we use two convolution layers and two hidden layers, we do not adopt the pooling layer to prevent performance degradation. The ReLU function is selected as an activation function. In the two convolution layers, 16 convolutional filters of sizes 8 and 4 are respectively used.

\subsection{Attack on Masked AES}

We also carry out a first order masked AES implementation on a XMEGA128 board. The 16 Sbox outputs are masked with the same masking value. Our second order attacks were performed using two identical attack steps using mask and MSbox output training data. The prior profiling of the mask and MSbox output value is completed during the training phase in the manner shown in Figure 6. 


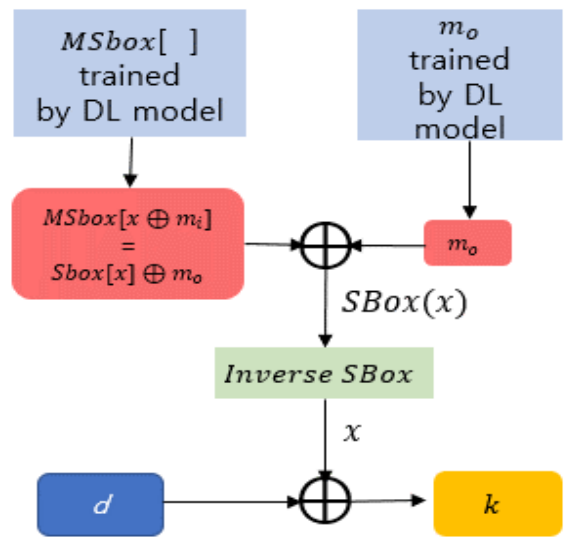

Figure 6: Second order attack using MSbox output and mask training data.

In the second order attack phase on first order masked AES, the mask training step is labeled the output mask mo. The 24,400 sample points of a trace during the following operation are used as inputs.

MSbox $\left[\right.$ index $\left.\oplus \mathrm{m}_{\mathrm{i}}\right]=\operatorname{Sbox}[\mathrm{index}] \oplus \mathrm{m}_{\mathrm{o}}$.

The number of output nodes is 256 , which is the range of a mask byte. In the MLP model, we respectively use two hidden layers and dropout layer with 0.25 dropout ratio. In the other $\mathrm{CNN}$ model, we use two convolution layers and two hidden layers, the same model used to attack an unprotected implementation. The main difference between the two models is the label used for data training. The previous label in the unprotected implementation is Sbox output for secret key recovery. On the other hand, the label for mask recovery is the masking value.

In the MSbox training phase, the label is the output value of M Sbox, that is, MSbox $\left[x \oplus m_{i}\right]=\operatorname{Sbox}(x) \oplus m_{0}$. The 600 sample points of a trace during masked SubBytes operation are used as inputs. When applying the MLP and CNN models, we use the same parameters as those described in the above mask training step.

Since the output mask $\mathrm{m}_{\mathrm{o}}$ and output of $\operatorname{MSbox}\left[\mathrm{x} \oplus \mathrm{m}_{\mathrm{i}}\right]$ are respectively recovered by the DL model, the output of Sbox can be computed as follows:

$\left.\operatorname{Sbox}(x)=\operatorname{MSbox}\left[x \oplus m_{i}\right] \oplus m_{0}=\left(\operatorname{Sbox}[x] \oplus m_{0}\right]\right) \oplus m_{0}$.

After computing the output of Sbox, the secret bytes of the initial AddRoundKey can also be recovered ( $\oplus$ Inverse Sbox $(\mathrm{x})$ ).

The accuracy evaluation of the correct key according to the number of epochs for each attack is described in Figure 7. In unprotected AES implementation, the adversary can extract the secret key with more than $98 \%$ accuracy in the case of 200 epochs. On the other hand, in masked implementation, the adversary can succeed with accuracies of $81.0 \%$ and $75.4 \%$ in the case of 300 epochs using the MLP and CNN models, respectively. Although it is not shown in Figure 7, the accuracies of mask recovery in the MLP and CNN models are all $100 \%$ in the case of 10 epochs. Here, the training time for each model takes less than about 1 minute.

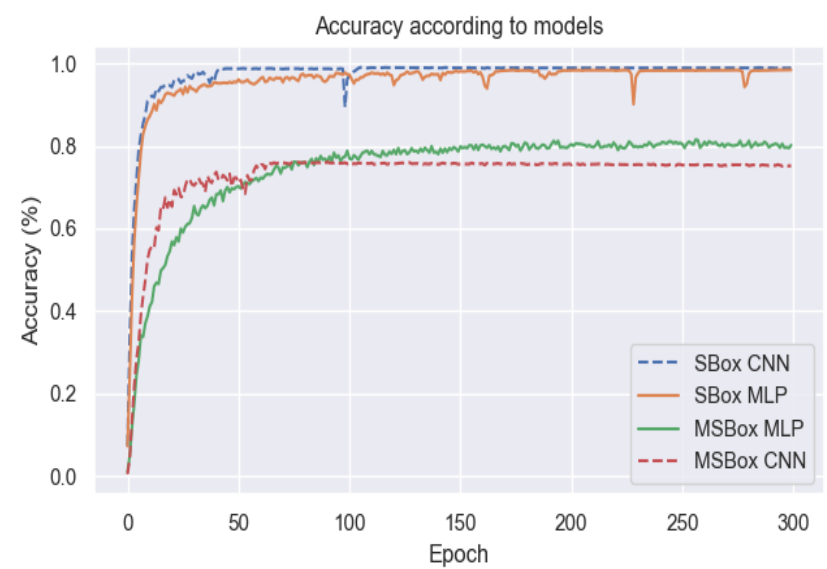

Figure 7: Accuracy of DL-based attack according to the number of epochs.

We now compare the accuracy of the DL-based side channel attack on AES implementation as shown in Table 1. First, we can observe that our DL-based attack can recover the secret key with an accuracy of over $98 \%$. This result is a significant improvement over several previous works on naïve AES implementation.

Table 1: Accuracy comparison of DL-based attacks.

\begin{tabular}{|l|l|l|l|}
\hline DL-based & \multirow{2}{*}{$\begin{array}{l}\text { Unprotected } \\
\text { Attack }\end{array}$} & \multicolumn{2}{|c|}{ Protected } \\
\cline { 3 - 4 } & Sbox $)$ & Mask & MSbox \\
\hline MLP[12 ] & 93.7 & - & - \\
\hline MLP[13] & 88.5 & & \\
\hline CNN[14] & 89.8 & - & - \\
\hline SVM+CPA[15] & - & 88.0 & - \\
\hline ANN[16] & - & 91.8 & 88.4 \\
\hline Our MLP & 98.4 & 100 & 81.0 \\
\hline Our CNN & 98.9 & 100 & 75.4 \\
\hline
\end{tabular}

In a previous attack described by Lerman et al. designed for protected implementation, the mask was recovered using an SVM (Support Vector Machine) with about $88 \%$ accuracy prior to performing a CPA (Correlation Power Analysis) attack. Our attack is composed of the recovery step of the mask with $100 \%$ accuracy and the recovery step of the masked Sbox output with accuracy ranging from about $75 \%$ to $81 \%$ according to use of the $\mathrm{CNN}$ or MLP model.

\section{CONCLUSIONS}

In this paper, we developed an efficient ML-based approach for evaluating the security level of a masked AES implementation. Our approach is composed of two profiling attacks, one targets the masking value and the other targets the masked intermediate value. Finally, it is shown that an adversary can extract the secret key by combining the recovered mask and the masked Sbox output value. The accuracy of the ML-based attack on protected AES implementation is as high as $81.0 \%$. 


\section{ACKNOWLEDGMENTS}

This work was supported by the National Research Foundation of Korea (NRF) grant funded by the Korean government (MSIT) (No. 2020R1F1A1074358).

\section{REFERENCES}

[1] Federal Information Processing Standards Publication (FIPS 197). 2001 Advanced Encryption Standard (AES). DIO: https://doi.org/10.6028/ NIST.FIPS. 197

[2] P. Kocher, J. Jaffe, and B. Jun. 1999. Differential Power Analysis. In Advances in Cryptology. M. Wiener (Eds.). Lecture Notes in Computer Science, Vol. 1666. Springer-Verlag, Berlin Heidelberg, 388-397. DIO: https://doi.org/10.1007/3-540-48405-1 25

[3] E. Brier, C. Clavier, and F. Olivier. 2004. Correlation Power Analysis with a Leakage Model. In International Workshop on Cryptographic Hardware and Embedded Systems. M. Joye, J. Quisquater (Eds.). Lecture Notes in Computer Science, Vol. 3156. Springer-Verlag, Berlin Heidelberg, 16-29. DIO: https://doi.org/10.1007/978-3-540-28632-5_2

[4] T. Messerges. 2001. Securing the AES finalists against power analysis attacks. In International Workshop on Fast Software Encryption (J. Hartmanis, J. Leeuwen, and B. Schneier (Eds.). Lecture Notes in Computer Science, Vol. 1978. Springer-Verlag, Berlin Heidelberg, 150-164. DIO: https://doi.org/10.1007/3-540-44706-7_11

[5] C. Herbst, E. Oswald, and S. Mangard. 2006. An AES smart card implementation resistant to power analysis Attacks. In International Conference on Applied Cryptography and Network Security. J. Zhou, M. Yung, and F. Bao (Eds.). Lecture Notes in Computer Science, Vol. 3989. Springer-Verlag, Berlin Heidelberg, 239-252. DIO: https://doi.org/10.1007/11767480_16

[6] E. Oswald and K. Schramm. 2005. An efficient masking scheme for AES software implementations. In International Workshop on Information Security Applications. E. Oswald and K. Schramm (Eds.). Lecture Notes in Computer Science, Vol. 3786. Springer-Verlag, Berlin Heidelberg, 292-305. DIO: https://doi.org/10.1007/11604938 23

[7] F. Rosenblatt. 1958. The perceptron: A probabilistic model for information storage and organization in the brain. Psychological Review, 65(6). 386-408. DOI: https://doi.org/10.1037/h0042519

[8] S. Albawi, T. A. Mohammed, and S. Al-Zawi. 2017. Understanding of a Convolutional Neural Network. In International Conference on Engineering and Technology (March 2018). 1-6. DOI: https://doi.org/ 10.1109/ICEngTechnol.2017.8308186

[9] ChipWhisperer - NewAE Technology Inc. 2017. Chipwhisperer. 2017 from http://newae.com/tools/ chipwhisperer/

[10] S. Chari, J. R. Rao, and P. Rohatgi. 2002. Template Attacks. In International Workshop on Cryptographic Hardware and Embedded Systems. B. Kaliski, K. Koç, and C. Paar (Eds.). Lecture Notes in Computer Science, Vol. 2523. Springer-Verlag, Berlin Heidelberg, 13-28. DIO: https://doi.org/10.1007/3540-36400-5_3

[11] W. Schindler, K. Lemke, and C. Paar. 2005. A Stochastic Model for Differential Side Channel Cryptanalysis. In International Workshop on Cryptographic Hardware and Embedded Systems. J. Rao and B. Sunar (Eds.). Lecture Notes in Computer Science, Vol. 3659. Springer-Verlag, Berlin Heidelberg, 30-46. DIO: https://doi.org/10.1007/11545262_3

[12] Z. Martinasek, and V. Zeman. 2013. Innovative method of the power analysis. Radioengineering, 22(2). 589-594. 2013 from http://www.radioeng.cz/fulltexts/2013/13_02_0586_0594.pdf

[13] H. Wang, M. Brisfors, S. Forsmark, and E. Dubrova. 2019. How Diversity Affects Deep-Learning Side-Channel Attacks. Cryptology ePrint Archive, Report 2019/664, 2019 from https://eprint.iacr.org/2019/664

[14] L. Wei, B. Luo, Y. Li, Y. Liu, and Q. Xu. 2018. I Know What You See: Power Side-Channel Attack on Convolutional Neural Network Accelerators. In Proceedings of the 34th Annual Computer Security Applications Conference (Dec. 2018). 393-406. DOI: https://doi.org/10.1145/ 3274694.3274696

[15] L. Lerman, S. F. Medeiros, G. Bontempi, and O. Markowitch. 2015. A machine learning approach against a masked AES. Journal of Cryptographic Engineering 5, 123-139. DIO: https://doi.org/10.1007/s13389-014-0089-3

[16] R. Gilmore, N. Hanley, and M. O'Neill. 2015. Neural network based attack on a masked implementation of AES, In IEEE International Symposium on Hardware Oriented Security and Trust. Washington, DC, USA. (July 2015), 106-111 DOI: https://doi.org/10.1109/HST.2015.7140247

[17] B. Gierlichs, L. Batina, P. Tuyls, and B. Preneel. 2008. Mutual information Analysis. In International Workshop on Cryptographic Hardware and Embedded Systems. E. Oswald and P. Rohatgi (Eds.). Lecture Notes in
Computer Science, Vol. 5154. Springer-Verlag, Berlin Heidelberg, 426-442. DIO: https://doi.org/10.1007/978-3-540-85053-3 